\title{
Perceived roughness as a function of body locus
}

\author{
JOSEPH C. STEVENS \\ John B. Pierce Foundation Laboratory and Yale University, New Haven, Connecticut
}

\begin{abstract}
Twenty subjects made magnitude estimates of the roughness of grooved metallic surfaces applied to 10 body loci. To a first approximation, perceived roughness grew as a power function of groove width, in accordance with earlier studies. The exponents and intercepts (up-down position in log-log coordinates) of the power function turn out to depend strongly on body locus. The straight lines in $\log$-log coordinates tend to diverge with groove width so that differences among body loci are especially pronounced at large groove widths. Sensitivity to roughness was greatest for the lips, fingers, and forearm, and least for the heel, back, and thigh. The rank order of the body loci in terms of roughness sensitivity closely parallels the rank order for punctate pressure sensitivity, as reported by von Frey in 1894, but apparently not for other measures of tactile sensitivity, such as vibration thresholds to various frequencies, two-point thresholds, and error of point localization.
\end{abstract}

Students of the psychophysics of the tactile sense have paid considerable attention to the question of how sensitivity varies over the body surface, well aware that the various body loci differ with respect to density of innervation as well as other properties of the skin, such as thickness, tautness, and temperature. Several kinds of measurements have been made. The first two go back to Weber (1834/1978)-namely, the two-point threshold and the error of point localization (as reported and confirmed experimentally over a century later by Weinstein, 1968). Next came the work of the sensory "spot mappers," beginning in the $1880 \mathrm{~s}$, and elaborated over the years. Data vis-a-vis body locus were assembled by von Skramlik in 1937 (see Woodworth \& Schlosberg, 1954). Another measure, first used by von Frey in 1894 (see Woodworth \& Schlosberg, 1954), is the threshold for punctate (hair) stimulation for both touch and pain (quite differently distributed for sensitivity over the skin surface). Thresholds of vibration at various frequencies and body loci also came under study by Wilska (1954). Finally, a much more recent approach (J. C. Stevens, 1979) examined sensitivity to weights of large area placed at various body loci. It turned out that such sensitivity correlated well with twopoint threshold and error of point localization, suggesting a common receptor network, but correlated with none of the other measures cited above (with the possible exception of spot density, for which there are too few loci mapped to make meaningful comparisons with the other studies).

The present study adds perceived roughness (a property of considerable interest to psychophysicists who study the

This research was supported by Grant NS15419 from the National Institutes of Health. The author thanks Cynthia Butler Leet for technical assistance, and Daniel Algom and Robert D. Melara for editorial suggestions. Correspondence may be addressed to Joseph C. Stevens, John B. Pierce Foundation Laboratory, 290 Congress Avenue, New Haven, CT 06519. skin) to the list of procedures. As will become clear, perceived roughness does depend on body locus, and apparently in much the same way as does punctate sensitivitybut on no other measure of regional sensitivity.

\section{METHOD}

\section{Stimuli}

The stimulators were six anodized aluminum alloy plates $(14 \mathrm{~cm}$ long, $3.7 \mathrm{~cm}$ wide, $2.5 \mathrm{~cm}$ thick) of the kind used by Green, Lederman, and J. C. Stevens (1979) and by Lederman and Taylor (1972), with parallel grooves cut rectangularly across the width of the plates. The only dimension that varied across the plates was the width of the grooves, which were, in the present experiment, $0.20,0.36$, $0.51,0.64,0.81$, and $1.02 \mathrm{~mm}$ wide. (These values are sometimes expressed below in inches in order to facilitate comparison with earlier studies and to conform to machinist specifications for manufacture.) The spacing among the grooves was constant at $0.25 \mathrm{~mm}$. Lederman (1974) and Lederman and Taylor (1972) have shown that groove width is the parameter of paramount interest when it comes to perceived roughness: the larger the groove width, the greater is the perceived roughness when the plates pass across the skin. Other parameters have only secondary bearing on the perception of roughness. These include the width of the "ridges" (lands), the velocity of travel across the skin, and pressure of contact (the most important of the secondary parameters) (see, e.g., Lederman, Loomis, \& Williams, 1982, and Lederman \& Taylor, 1972). Various studies have shown that estimated roughness exhibits the power law of sensory magnitude (S. S. Stevens, 1975).

\footnotetext{
Subjects

The subjects were 10 men and 10 women, whose mean age was 25 years (range 18-37 years). Each subject took part in a test session that lasted approximately $45 \mathrm{~min}$. The subject, clad in shorts and shirt, lay on a mattressed surface, at an elevation that facilitated application of the stimulators. From time to time, the subject had to turn from supine to prone position, or vice versa, to permit stimulation of the various loci chosen for study. These were, in order of stimulation, forearm (ventral), fingertips, belly, thigh, heel, forehead, lips, upper arm (dorsal), back (midriff), and calf. In the course of testing, each of these 10 areas was stimulated twice by each of the six blocks, in random order with respect to groove width. The perceived roughness magnitude was judged by the method of
} 
free magnitude estimation (no designated standard or modulus; S. S. Stevens, 1975). This means that each subject made 120 magnitude estimates.

\section{Procedure}

On each trial, the experimenter lightly stroked the skin twice for about $1 \mathrm{sec}$ per stroke, separated by about $1 \mathrm{sec}$. The stroke was always delivered at right angles to the longitudinal plane of the body. Every effort was made to make the strokes as uniform as possible with respect to velocity and pressure; the work mentioned above by Lederman and colleagues implies that minor variations on these dimensions would matter little, if at all.

At the start of the session, the experimenter instructed the subject that the task was to judge how rough various metal plates felt when rubbed over different parts of the body. One of the plates was then demonstrated by stroking it across the arm of the experimenter. The subject was to assign a number deemed appropriate to stand for the experienced roughness on the first application $(0.51-\mathrm{mm}$ groove width to the ventral forearm) and thereafter to assign numbers proportional to the perceived degree of roughness. Thus, if the first number assigned was, say, 10, and the second stimulus felt one fourth as rough, it was to receive the number 2.5, and so forth. Fractions and decimals were permitted when needed. The number assigned to the first application was not counted in the data analysis. Instead, the $0.51-\mathrm{mm}$ stimulus was presented to the forearm three times, but only the second and third presentations counted. A judgment of zero would mean the plate felt absolutely smooth. This was reported a total of 28 times by 5 subjects, mostly, but not exclusively, for the narrowest groove width and most prominently for the heel.

The subjects gave informed consent and were paid $\$ 5$ for their participation.

\section{RESULTS}

Because the subjects operated on various regions of the number continuum, given the freedom to choose any number to stand for the roughness of the first presentation, it was necessary to normalize the data by a procedure like that first suggested by Lane, Catania, and S. S. Stevens (1961) and used routinely since then. The grand mean of each subject's 120 estimations was calculated and multiplied by a factor, $c$, that made the resulting product equal 10 (an arbitrary but convenient number). Then all of the subject's estimates were multiplied by the normalization factor $c$. The normalized estimates were then averaged arithmetically across subjects. This procedure eliminates arbitrary differences among subjects' choices of the portion of the number continuum on which to operate.

Because magnitude estimates are often distributed approximately log-normally, it is customary and desirable to average them geometrically (J. C. Stevens, 1957; S. S. Stevens, 1975). In the present case, as in many others, this proved impossible because of the occurrence of a considerable number of zero estimates. The occurrence of zero estimates has been a recurrent headache in the analysis of magnitude estimates. For this Icason, it may be instructive to detail how the problem yielded to solution in the present case (and potentially to many others). First, the normalization process greatly reduces the variability of the estimates, without altering their relative values, thereby greatly reducing the difference between the arith- metic and geometric means. Besides, of the 45 combinations of groove width and body locus that drew no zero responses (out of 60 combinations altogether), the relation between the arithmetic and geometric mean is close to a simple proportionality, as shown in Figure 1. In other words, the arithmetic means are, throughout, a nearly constant $7.5 \%$ larger than the geometric means. As will be evident below, this difference has no significant bearing on the interpretation of the outcome.

Figure 2 shows, for each of the 10 body loci, how the mean estimate of roughness depends on groove width (lower $\mathrm{x}$ axis in inches, upper $\mathrm{x}$ axis in millimeters). The data for body loci are plotted separately because when plotted together, overlap of points from one locus to another obscures the form and fit of the individual functions. That the data are, in general, fitted well by straight lines in log-log coordinates signifies that perceived roughness, $y$, approximates a power function of groove width, $x$, such that

$$
\log y=\beta \log x+\log k,
$$

or, taking the antilogarithms of Equation 1,

$$
y=k x^{\theta} .
$$

The constants $\beta$ and $\log k$ are listed in Table 1 for the 10 body loci, together with the Pearson correlation $r$ relating the $\log y$ point values and the $\log x$ point values as an indication of the goodness of fit of the power function. Except for the heel and the back, these coefficients are high. Discrimination of roughness level via the heel and back thus appears to have been quite difficult. In view of the very large scatter (low $r$ ) for the heel, one should be wary of concluding that its $\beta$, seemingly out of line with the others, is really significantly different.

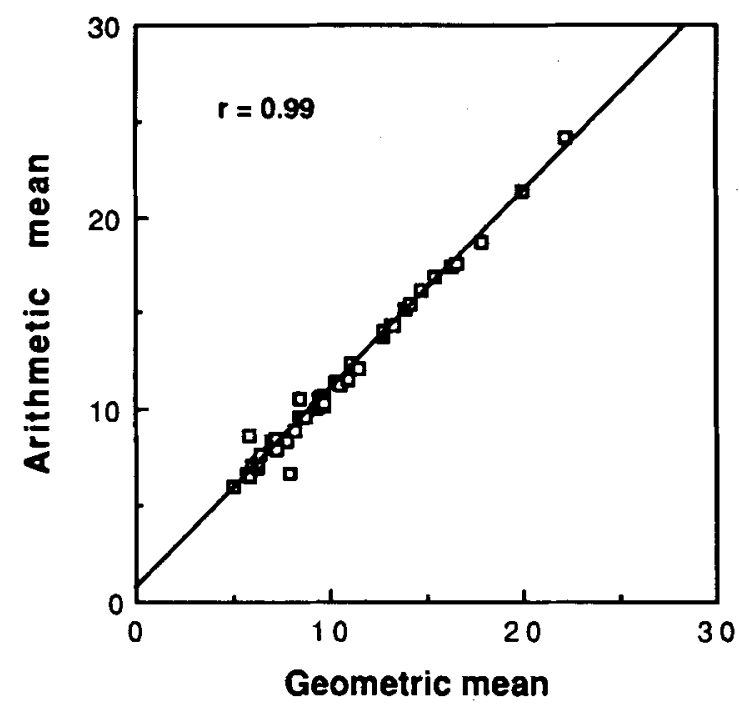

Figure 1. Arithmetic means as a function of the geometric means of the magnitude estimates of the $\mathbf{4 5}$ combinations of groove width and body locus that drew no zero estimates. 


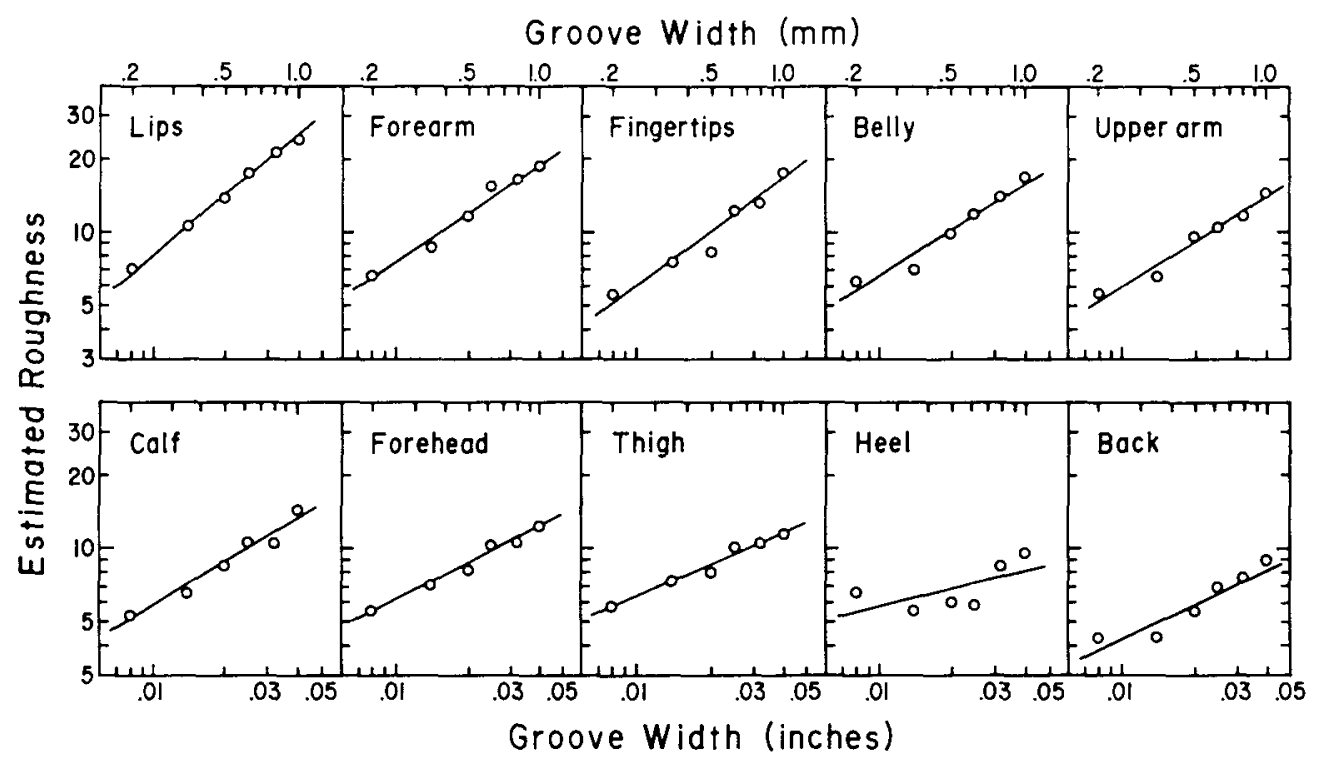

Figure 2. Arithmetic means of the normalized estimates of roughness as a function of groove width in inches (lower $x$ axis) and millimeters (upper $x$ axis). The 10 functions for 10 body loci were fitted by the method of least squares, operating on the logarithms of both the $x$ and the $y$ values.

Note that the values of $\beta$ in Table 1 would be nearly the same whether they are based on arithmetic or geometric means, despite (or because of) the finding that the arithmetic means are a constant percentage larger than the geometric means. That is, multiplication of all of the $y$ points by a constant has no effect on the exponent (slope) of a power function.

Power functions like those in Figure 2 were reported earlier by Green, Lederman, and J. C. Stevens (1979) and by Lederman and Taylor (1972) for active fingertip stimulation by means of the kind of grooved plates used in the present experiment. The exponents from those earlier studies were substantially larger than those reported here-on the order of 1.0 or larger. Differences in

Table 1

$\beta$ and $\log k$ Constants (for Inches and Millimeters) of the Power Functions Relating Mean Normalized Magnitude Estimates of Roughness to Groove Width in Inches, Together With the Pearson Correlation Coefficient $r$ Relating the $x$ Points and the $y$ Points as an Indicant of Goodness of Fit

\begin{tabular}{lllll}
\hline \multicolumn{1}{c}{ Locus } & $\beta$ & $\begin{array}{c}\log k \\
\text { (in.) }\end{array}$ & $\begin{array}{c}\log k \\
\text { (mm) }\end{array}$ & $r$ \\
\hline Lips & .78 & 2.48 & 1.38 & .998 \\
Forearm & .66 & 2.21 & 1.27 & .987 \\
Fingers & .73 & 2.25 & 1.22 & .974 \\
Belly & .63 & 2.08 & 1.19 & .966 \\
Upper arm & .59 & 1.97 & 1.13 & .978 \\
Calf & .60 & 1.96 & 1.11 & .975 \\
Forehead & .49 & 1.78 & 1.08 & .991 \\
Thigh & .43 & 1.66 & 1.06 & .982 \\
Back & .44 & 1.51 & 0.88 & .913 \\
Heel & .24 & 1.25 & 0.90 & .632 \\
\hline
\end{tabular}

Note-The values of $\beta$ are the same whether groove width is expressed in inches or millimeters. procedural detail may account for this. For example, the earlier studies used active touch, the present study used passive touch (this particular explanation seems unlikely in view of Lederman's, 1981, report of little difference between roughness aroused actively and passively). It has been the experience of many investigators that when subjects find a psychophysical scaling task to be difficultand the present one surely was-they tend to restrict the number range emitted and thereby markedly flatten the psychophysical function (Algom \& Marks, 1984; Cafarelli, Cain, \& J. C. Stevens, 1977; Marks, 1968; J. C. Stevens, 1974b; J. C. Stevens \& Cain, 1970; J. C. Stevens \& Hall, 1966; J. C. Stevens \& Krimsley, 1977; S. S. Stevens \& Greenbaum, 1966). Ways in which magnitude estimation becomes more difficult include requiring the subject to estimate more than one perceived dimension of the sensation or to estimate the perceived magnitude of stimuli that vary along more than one physical dimension.

Another possible consideration regarding slope is that Lederman (personal communication, August 1989) has sometimes fitted a two-limbed psychophysical function to roughness data. There is some evidence for a twolimbed function in 7 of the 10 panels of Figure 2, and six subsequently measured (as yet unpublished) functions for the finger also showed a clear difference between the slopes of the upper and lower limbs. For the present data set, however, the overall fit to simple power functions provides a useful description.

Whatever the explanation for the relatively flat functions here, it is important to recognize that what matters in experiments employing magnitude estimation with more than one stimulus or response dimension is the relative 
sizes of the slopes and vertical positions of the functions in $\log$ - $\log$ coordinates.

To get the overall picture, it is instructive to plot in the same $\log -\log$ axes (Figure 3 ) all 10 power functions as given by the constants listed in Table 1 . Two main features emerge from this graph with regard to regional differences in the perception of roughness. The first is that, to some body loci, the same groove widths feel considerably rougher than to others. The lips, forearm, and fingers are especially sensitive; the heel, back, and thigh especially insensitive. The second feature is the tendency of the functions to converge toward small groove widths. That is, the body surface tends to have more uniform sensitivity to stimuli that are relatively less rough.

When the lines in Figure 3 are extended toward very small stimulus values, they fail to converge exactly at a common point, but they come close enough to suggest that if there were no "noise" in the functions depicted in Figure 2, they might converge in the vicinity of a hypothetical groove width of $0.13 \mathrm{~mm}(0.0046 \mathrm{in}$.). This argument is based on a proof given by J. C. Stevens (1974a) and J. C. Stevens and Rubin (1970; see also Algom \& Cohen-Raz, 1984; Luce \& Krumhansl, 1988). If a family of power functions does converge at a low stimulus level, the exponent $\beta$ must be a linear function of $\log k$. Assume that the family of functions in Figure 2 does ideally converge on a common point, $\log y=-a$ and $\log x=-c$. Then by substitution and rearrangement of Equation 1,

$$
c \beta=\log k+a \text {. }
$$

Figure 4 is a plot of the slopes as a function of the $\log k s$ obtained by least squares, as shown in Table 1 . The func-

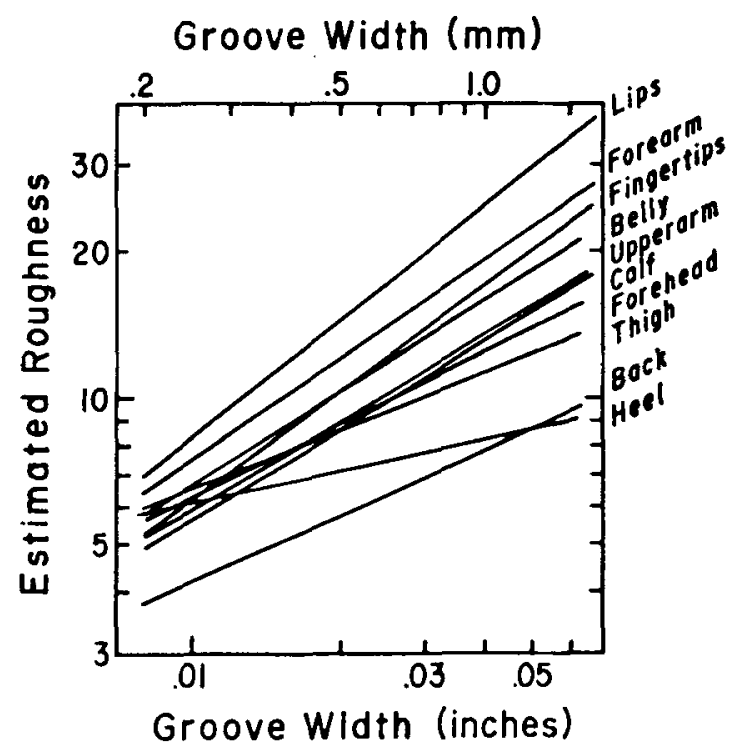

Figure 3. Power functions for 10 body loci all plotted in the same $\log -\log$ axes. These functions are identical in slope and intercept to those shown in Figure 2.

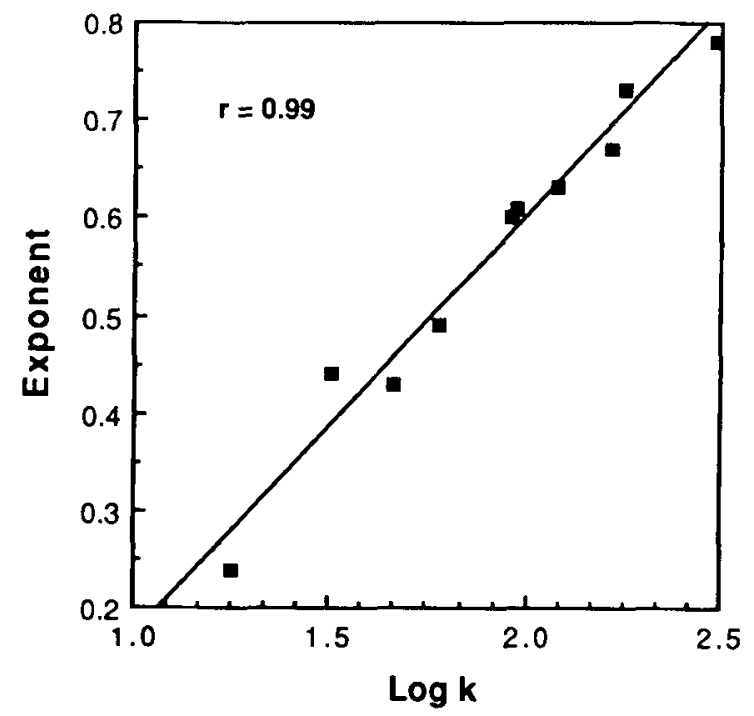

Figure 4. The exponent $\beta$ plotted as a function of the intercept $\log k$. The line was fitted by the method of least squares.

tion is indeed linear to a first approximation, and the Pearson coefficient $r$ relating the $y$ and $x$ values of the points in Figure 4 equals .99.

To solve for the "ideal" intersection point $x_{i}$, one takes any two points on the function in Figure 4, such that

$$
k_{1} x_{1}^{\beta_{1}}=k_{2} x^{\beta_{2}},
$$

and by rearrangement of terms,

$$
x_{i}=\left(\log k_{1}-\log k_{2}\right) /\left(\beta_{2}-\beta_{1}\right),
$$

which is the negative of the slope of the function in Figure 4 . This value turns out to be $0.13 \mathrm{~mm}(0.0046 \mathrm{in}$.). It remains to be determined whether this value has empirical significance-for example, whether it might signify that groove width that is perceived as completely smooth to all sites on the skin. For other families of converging power functions, the convergence point often yielded intuitively to empirical interpretation. One example is the psychophysical function for the perceived heaviness of lifted weights that varied in both mass and volume; the convergence point corresponded closely to the maximum heft possible under the test conditions ( $J$. C. Stevens \& Rubin, 1970). Another example is the psychophysical functions for apparent warmth as a function of flux density of infrared radiation over various areal extents of the skin; these functions show that the skin integrates the effects of radiation over area, but does so less and less completely as the level of flux density increases. The psychophysical functions in this case converge on the absolute threshold of pain, which is notable for its lack of spatial summation (Marks \& J. C. Stevens, 1973; J. C. Stevens \& Marks, 1971; J. C. Stevens, Marks, \& Simonson, 1974).

Finally, the functions in Figure 3 can be used to construct equal-sensation profiles of the skin-that is, to determine the groove widths needed to arouse the same 
degree of perceived roughness for all body sites. To do this, one makes a horizontal cut through Figure 3 at any desired roughness level and reads from the graph the $x$ value corresponding to each body locus. Alternatively, one can use the power-function constants in Table 1 to solve for all the $x$ values that make a given level of $y$ constant. As an illustration, we solved the equations for $y$ $=5,7.5,10,15$, and 20, and these are shown in Figure 5 . Here we see in another way how sensitivity to roughness varies from one locus to another. Of course, the profile varies with the level of $y$. At higher levels of $y$, the profiles appear "steeper," at lower levels, "flatter." This reflects the fact that regional differences are greater at high levels than at low levels.

An interesting feature of equal-sensation profiles (and many other families of equal sensation) is that they are independent of the absolute sizes of the exponents and intercepts of the psychophysical functions used to generate them. It is necessary to know only the relative sizes of these constants. Hence, if all the exponents in Table 1 were multiplied by a constant to bring them into better conformity with earlier reported exponents, that would in no way alter the shape of the profile shown in Figure 5.

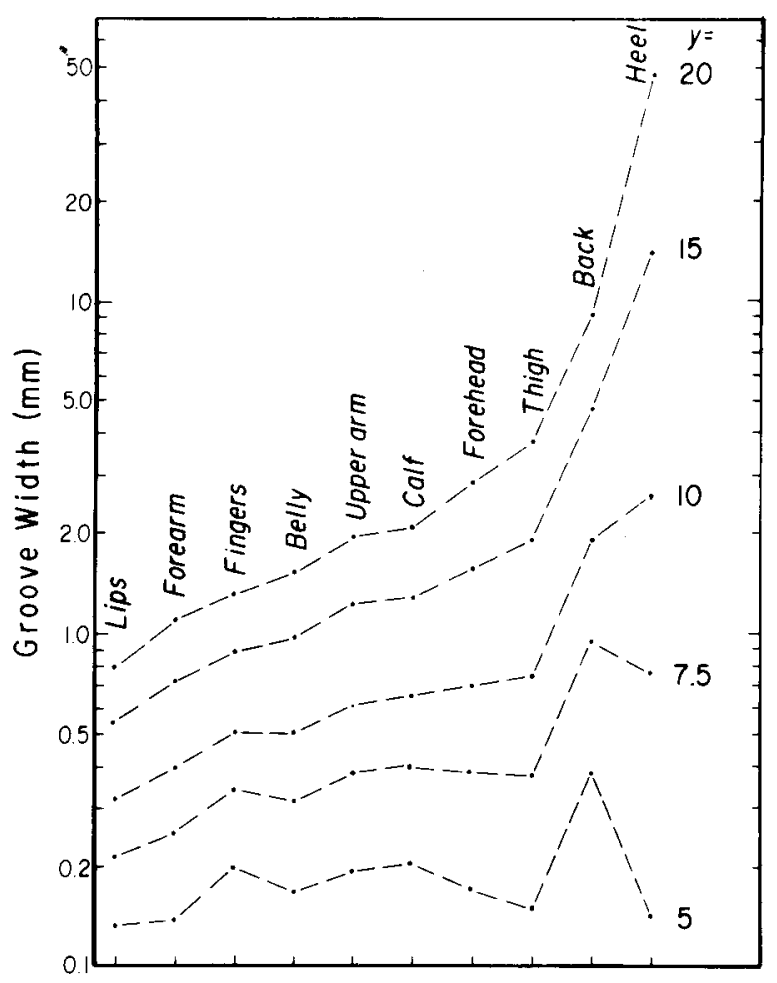

Figure 5. Five profiles of sensitivity of the 10 body regions, constructed from the constants in Table 1 . This graph shows the groove width (logarithmic ordinate) necessary to arouse five constant degrees of perceived roughness, $y$, as in Equation $2(y=5,7.5,10,15$, and 20 in Figure 3).

\section{DISCUSSION}

The results agree with earlier studies that the perceived degree of roughness can be characterized by a power function of the groove width of metallic plates. What is new here is that the exponent (slope) and the up-down position, as reflected by the value of $\log k$ in Equation 1 , depend considerably on the body locus stimulated. For example, the plate having the largest groove width $(1.02 \mathrm{~mm})$ was judged on the average to feel about three times rougher to the lips than to the back. Such regional differences tend, however, to be smaller for smaller groove widths.

It is instructive to compare how regional sensitivity to roughness correlates with several other kinds of cutaneous sensitivity, such as two-point threshold (Spearman $\varrho$ $=.41, p<.25,10$ body regions as measured by Weinstein, 1968), error of point localization $(\varrho=.55$, $p<.10,10$ regions as measured by Weinstein, 1968), and weight sensitivity ( $\rho=.90, p<.40$, five body regions as measured by J. C. Stevens, 1979) (all three of these measures correlate well with one another), punctate pressure sensitivity (see following paragraph) and pain thresholds, and vibration thresholds over a wide frequency range $(\varrho=-.033, p<.93$, nine regions for $50 \mathrm{~Hz}$ as measured by Wilska, 1954; $e=.22, p<.58$, nine regions for $400 \mathrm{~Hz}$ as measured by Wilska, 1954).

The only one of these sensitivities that correlates really well with roughness sensitivity appears to be punctate pressure sensitivity as measured by von Frey in 1894 for seven of the body regions examined in the present study (as reported by Woodworth \& Schlosberg, 1954). The measure of roughness sensitivity taken was the mean of the normalized estimate of the widest groove width $(1.02 \mathrm{~mm})$. It was chosen because this stimulus drew no zero estimates and appeared to be quite "stable." The rank-order correlation between punctate pressure and roughness sensitivities (Spearman e) equals .93 $(p<.003)$. Such high correlation indicates that punctate pressure sensitivity and roughness could be mediated by the same receptor network, a possibility that those who study innervation of the skin might want to keep in mind.

For at least two reasons, however, it would be premature to conclude that the regional differences shown by the two measures are fully explanable by regional differences in the density of innervation by a certain type of receptor (possibly Meissner corpuscles, which were strongly implicated by LaMotte, 1977, in the mediation of roughness by fabrics).

First, there exists the possibility that various types of receptors cooperate in the mediation of roughness. In a relevant physiological study, Goodwin, John, Sathian, and Darian-Smith (1989) simulated human tactual exploration by moving alternating grooves and ridges sinusoidally over the monkey's fingerpad while recording neural responses of three afferent types: rapidly adapting (RA), 
slowly adapting (SA), and Pacinian (PC). All three types yielded an increasing response as a (power) function of groove width, given constant ridge width. For RAs and PCs, the response was linear with groove width (exponent - 1.0); for SAs it was an accelerated function of groove width (exponent $\sim 2.6$ ).

Second, regional differences in mechanical properties of the skin may also count. The thick epidermis of the sole and the heel, for example, would seem likely to matter. As it stands, therefore, we have insufficient knowledge to explain the psychophysical results in terms of neural and anatomical properties. It remains, however, an intriguing question why punctate sensitivity and roughness sensitivity appear to correlate so well with each other and seemingly very poorly, if at all, with other measures of tactile sensitivity.

It comes as no surprise that the fingertips and the lips have high punctate and roughness sensitivity, given their critical roles in the exploration of the tactile world (in the case of the lips, especially in young childhood). Less easy to understand is the relatively high sensitivity of the ventral forearm. In general, it may be said that it is somewhat surprising that although regional differences are marked, they seem loosely matched to their apparent "use" in perceiving the tactile world. Perhaps the explanation (admittedly highly speculative) has to do with the fact that unlike the furry mammals, human beings have to protect and thermoregulate the integument with clothing, which can sooth or irritate the skin, depending on fabric characteristics (Gwosdow, J. C. Stevens, Berglund, \& Stolwijk, 1986). Or alternatively, the receptor system appealed to by punctate and roughness sensitivity may be tuned to register the presence of stationary and moving touch sensation somewhere on the skin, rather than its exact spatial locus and/or distribution. In any case, the present outcome seems to be counter to the hypothesis that local differences in two-point threshold can predict local differences in dynamic pattern recognition (Loomis \& Lederman, 1986).

In contrast, the regional distribution of tactile spatial acuity, as gauged by two-point discrimination and error of localization, seems to be tightly matched to tactile exploration. These measures vary greatly from one body site to another. Thus, the trunk and lower limbs have extremely poor acuity compared with the face and the distal portions of the upper limbs. And along the arm, acuity declines steadily and precipitously from fingertips to shoulder, often called Vierordt's (1870) law of mobility. This law argues that the greater the flexibility of movement of a body part, the greater is its acuity. It would seem, then, that tactile acuity and punctate and roughness sensitivity play quite different roles in the perception of the tactile world, even though the details are conjectural.

Finally, the present results leave unresolved the relative roles of static and dynamic components of the stimulation for perceived roughness. Plainly, spatial periods of the type of gratings used here, although effective in arousing roughness sensation, are too small to resolve on stationary contact. This difference stands out in a region like the forearm, which responds well to roughness but relatively unimpressively to static patterns, such as those provided by the two-point or two-edge esthesiometer, and to square-wave gratings of the kind used by Johnson and Phillips (1981) to study static resolution. Experimentation that manages to bridge the gap between static and dynamic stimulus modes might help to elucidate the perceptual differences between static acuity and perceived roughness.

\section{REFERENCES}

Algom, D. , Cohen-Raz, L. (1984). Visual velocity input-output functions: The integration of distance and duration onto subjective velocity. Joumal of Experimental Psychology: Human Perception \& Performance, 10, 486-501.

Algom, D., \& MARKS, L. E. (1984). Individual differences in loudness processing and loudness scales. Journal of Experimental Psychology: General, 113, 571-593.

Cafarelli, E. Cain, W. S., Stevens, J. C. (1977). Effort of dynamic exercise: Influence of load, duration, and task. Ergonomics, 20, 147-158.

Goodwin, A. W., John, K. T., Sathian, K. d Darian-Smith, I. (1989). Spatial and temporal factors determining afferent fiber responses to a grating moving sinusoidally over the monkey's fingerpad. Journal of Neuroscience, 9, 1280-1293.

Green, B. G., Lederman, S. J., \& Stevens, J. C. (1979). The effect of skin temperature on the perception of roughness. Sensory Processes, 3, 327-333.

Gwosdow, A. R., Stevens, J. C., Berglund, L. G., \&tolwuk, J. A. J. (1986). Skin friction and fabric sensations in neutral and warm environments. Textile Research Journal, 56, 574-580.

Johnson, K. O., \& PhillJPs, J. R. (1981). Tactile spatial resolution: I. Two-point discrimination, gap detection, grating resolution, and letter recognition. Journal of Neurophysiology, 46, 1177-1191.

LAMotTE, R. H. (1977). Psychophysical and neurophysiological studies of tactile sensibility. In N. R. S. Hollies \& R. F. Goldman (Eds.), Clothing comfort (pp. 83-105). Ann Arbor, MI: Science Publishers.

Lane, H. L., Catania, A. C., Stevens, S. S. (1961). Voice level: Autophonic scale, perceived loudness and effects of sidetone. Journal of the Acoustical Society of America, 33, 160-167.

LEDERMAN, S. J. (1974). Tactile roughness of grooved surfaces: The touching process and effects of macro- and microsurface structure. Perception \& Psychophysics, 16, 385-395.

LEDERMAN, S. J. (1981). The perception of surface roughness by active and passive touch. Bulletin of the Psychonomic Society, 18, 253-255.

Lederman, S. J., Loomis, J. M., \& Williams, D. A. (1982). The role of vibration in the tactual perception of roughness. Perception \& Psychophysics, 32, 109-116.

Lederman, S. J., TAYLOR, M. M. (1972). Fingertip force, surface geometry, and the perception of roughness by active touch. Perception \& Psychophysics, 12, 401-408.

LoOMis, J. M., \& LedermaN, S. J. (1986). Tactual perception. In K. R. Boff, L. Kaufman, \& J. P. Thomas (Eds.), Handbook of perception and human performance (chap. 31, pp. 1-41). New York: Wiley.

LuCE, R. D., \& KrumhansL, C. L. (1988). Measurement, scaling and psychophysics. In R. C. Atkinson, R. J. Herrnstein, G. Lindzey, \& R. D. Luce (Eds.), Stevens' handbook of experimental psychology (2nd ed., Vol. 1., pp. 287-288). New York: Wiley.

MARKS, L. E. (1968). Stimulus-range, number of categories, and form of the category-scale. American Joumal of Psychology, 81, 467-479.

MARKS, L. E., \& STEvens, J. C. (1973). Spatial summation of warmth: Influence of duration and configuration of the stimulus. American Jour nal of Psychology, 86, 251-267. 
STEVENS, J. C. (1957). A comparison of ratio scales and category scales for the loudness of white noise and the brightness of white light. Unpublished doctoral dissertation, Harvard University, Cambridge, MA.

Stevens, J. C. (1974a). Families of converging power functions in psychophysics. In H. R. Moskowitz, B. Scharf, \& J. C. Stevens (Eds.), Sensation and measurement: Papers in honor of S. S. Stevens (pp. 157-165). Dordrecht, Holland: Reidel.

STEveNs, J. C. (1974b). Psychophysical invariances in proprioception. In F. A. Geldard (Ed.), Cutaneous communication systems and devices (pp. 73-77). Austin, TX: Psychonomic Society.

Stevens, J. C. (1979). Thermal intensification of touch sensation: Further extensions of the Weber phenomenon. Sensory Processes, 3, 240-248.

Stevens, J. C., Cain, W. S. (1970). Effort in isometric muscular contractions related to force level and duration. Perception \& Psychophysics, 8, 240-244.

Stevens, J. C., \& Hall, J. W. (1966). Brightness and loudness as functions of stimulus duration. Perception \& Psychophysics, 1, 319-327.

Stevens, J. C., \& Krimsley, A. L. (1977). Buildup of fatigue in static work: Role of blood flow. In G. Borg (Ed.), Physical work and effort (pp. 145-155). Oxford: Pergamon.

Stevens, J. C., \& Marks, L. E. (1971). Spatial summation and the dynamics of warmth sensation. Perception \& Psychophysics, 9, 391-398.

Stevens, J. C., Marks, L. E., \& Simonson, D. (1974). Regional sen- sitivity and spatial summation in the warmth sense. Physiology \& Behavior, 13, 825-836.

Stevens, J. C., \& Rubin, L. L. (1970). Psychophysical scales of apparent heaviness and the size-weight illusion. Perception \& Psychophysics, 8, 225-230.

Stevens, S. S. (1975). Psychophysics: Introduction to its perceptual, neural and social prospects. New York: Wiley.

Stevens, S. S., \& Greenbaum, H. B. (1966). Regression effect in psychophysical judgment. Perception \& Psychophysics, 1, 439-446.

VIERORDT, K. (1870). Die Abhängigkeit der Ausbildung des Raumsinnes der Haut von den Beweglichkeit der Körperteile. Zeitschrift für Biologie, 6, 53-72.

WEBER, E. H. (1978). The sense of touch (H. E. Ross \& D. J. Murray, Trans.). London: Academic Press. (Original work published 1834)

WeINSTEIN, S. (1968). Intensive and extensive aspects of tactile sensitivity as a function of body part, sex, and laterality. In D. R. Kenshalo (Ed.), The skin senses (pp. 195-222). Springfield, IL: Thomas.

WILSKA, A. (1954). On the vibrational sensitivity in different regions of the body. Acta Physiologica Scandinavica, 31, 285-289.

WoODWORTH, R. S., \& Schlosberg, H. (1954). Experimental psychology (rev. ed.). New York: Holt, Rinehart, \& Winston.

(Manuscript received June 19, 1989;

revision accepted for publication September 15, 1989.) 